
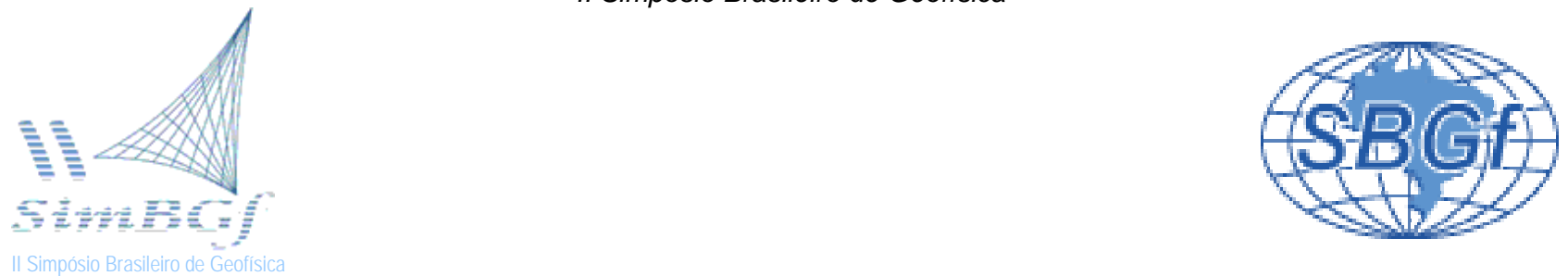

21 a 23 de Setembro de 2006, Natal - RN

\title{
Previsão probabilística de grandes perturbações geomagnéticas
}

\author{
Vitor H. A. Dias ${ }^{1,2}$, Jorge O. O. Franco ${ }^{1,2}$, Lílian P. Sosman ${ }^{2}$ e Andrés R. R. Papa ${ }^{1,2}$, ON/MCT e UERJ.
}

Copyright 2004, SBGf - Sociedade Brasileira de Geofísica

Este texto foi preparado para a apresentação no I Simpósio de Geofísica da Sociedade Brasileira de Geofísica, São Paulo, 26-28 de setembro de 2004. Seu conteúdo foi revisado pela Comissão Técnico-científica do I SR-SBGf mas não necessariamente representa a opinião da SBGf ou de seus associados. É proibida a reprodução total ou parcial deste material com propósitos comerciais sem prévia autorização da SBGf.

\section{Resumo}

Neste trabalho estudamos a possibilidade de previsão de perturbações e tempestades magnéticas. Ela é importante pois alguns métodos de prospecção e perfuração de poços estão baseados nos valores ou orientação do campo magnético terrestre. Desvios dos valores normais podem levar à não consecução dos objetivos propostos. Se faz um estudo das flutuações do índice Sym-H (global) e de medições obtidas no Observatório de Vassouras (regional), que pode vir a ter aplicação para as atividades em território brasileiro.

\section{Introdução}

Existem alguns métodos geofísicos para os quais o acontecimento de tormentas ou tempestades magnéticas é desejado e útil, por exemplo, MT. Para muitos outros, tais como medições do campo magnético crustal da Terra com objetivo de prospecção, as perturbações geradas na ionosfera e magnetosfera são uma fonte de erro, limitando potencialmente a exatidão das medições [Gleisner et al., 2005]. Na perfuração direcional, o campo geomagnético é usado para monitorar a direção em que o poço é aberto, é suposto como conhecido e usado como referência [Gleisner et al., 2005]. Qualquer desvio dos níveis não perturbados pode causar erros de referência levando a afastamentos da direção de perfuração pretendida. Nas atividades deste tipo no Mar do Norte, são consideradas perturbações de "grande" magnitude se: $\Delta F>145 n T, \Delta D>22$ ' e $\Delta I>10$ '; onde $F$ é a intensidade do campo, D a declinação e I a inclinação, e $\Delta \mathrm{F}, \Delta \mathrm{D}$ e $\Delta \mathrm{I}$ são os valores absolutos da perturbação em cada um deles [Gleisner et al., 2005]. Por tudo o anterior (e outras muitas razões, p.e., a saúde humana [Halberg et al., 2001]) seria muito interessante se existissem métodos de prever as maiores perturbações com suficiente antecedência. Mas ainda não existem métodos que permitam essa previsão de modo confiável.

\section{Seleção de dados}

O índice Dst (com resolução temporal de 1 hora), que é essencialmente o mesmo que o Sym-H (com resolução temporal de 1 minuto), é normalmente usado como testemunho do acontecimento de tormentas ou tempestades magnéticas. Freqüentemente é tomado o valor $-50 \mathrm{nT}$ desse índice como limiar para considerar que uma tormenta magnética está a acontecer [Maltsev, 2003]. Na Figura 1 apresentamos as variações do índice Dst para o mês de outubro de 2000. Os dados foram obtidos do World Data Centre for Geomagnetism (WDC), em Kyoto, Japão.

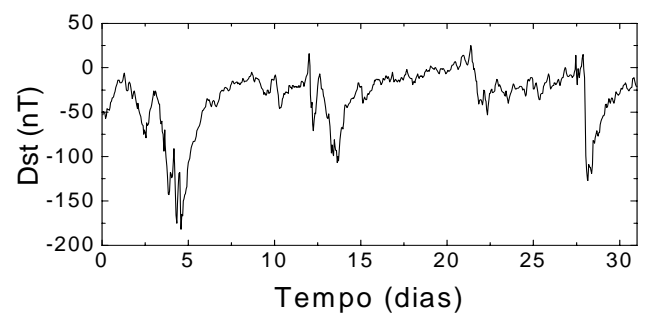

Figura 1 - Dependência temporal do índice Dst para o mês de outubro de 2000.

Analisando a Figura 1 e outros períodos de um mês (que não mostramos aqui), pode se ver que existem duas características que estão presentes em um número considerável de casos: uma repentina diminuição nos valores do índice Ds (durante umas poucas horas) e uma lenta recuperação (durante dois ou três dias) para valores próximos de zero. Nessa característica foi baseada a nossa escolha de dados. Ela consistiu em um conjunto de passos relativamente simples que passamos a descrever agora. Usamos como critério de limiar para o começo de uma tormenta magnética o valor $-50 \mathrm{nT}$ do índice Dst. Com isto, localizamos a data e hora de todas as tormentas magnéticas no período 1998-2002. O começo do transiente antes das tormentas foi estimado como aquele em que se obteve um valor maior que -10 nT pela última vez antes da tormenta. Não consideramos transientes maiores que 10 horas (e a correspondente tormenta não foi incluída no conjunto a ser estudado). O valor médio desses períodos foi de 6 horas e foi tomado como único para todas as tormentas. Também eliminamos da nossa seleção todas aquelas tormentas muito próximas de tormentas anteriores, pela impossibilidade de se ter, para elas, um período "limpo" de seis dias mais seis horas de duração (veja a explicação a seguir). O resultado final foi que eliminamos ao redor de $50 \%$ dos períodos inicialmente selecionados (60 casos). Nós investigamos duas janelas de tempo de três dias: os três dias imediatamente anteriores ao começo do transiente e os três dias imediatamente anteriores a estes. Três dias é considerado um período 
razoável para a previsão deste tipo de fenômeno [Gleisner et al., 2005].

\section{Índice Sym-H}

Os valores aqui estudados do índice Sym-H foram obtidos do World Data Centre for Geomagnetism, em Kyoto, Japão. Os resultados que apresentamos correspondem ao período de janeiro de 1998 até dezembro de 2002. A Figura 2 mostra as distribuições dos valores do índice Sym-H para o mês de outubro de 2000. Ela segue uma lei de potências do tipo:

$$
f(x)=k \cdot x^{d}
$$

onde $f(x)$ é a distribuição de freqüências da variável $x, k$ é uma constante de proporcionalidade e $d$ é o expoente da lei de potências.

Inicialmente estudaremos a variação de $d$ de um tipo de janela temporal para o outro. Foram calculadas as distribuições dos valores do índice Sym-H para cada tipo de período. Note, contudo, que esta é uma estratégia perigosa pois teremos amostras menores e, conseqüentemente, uma pior estatística.

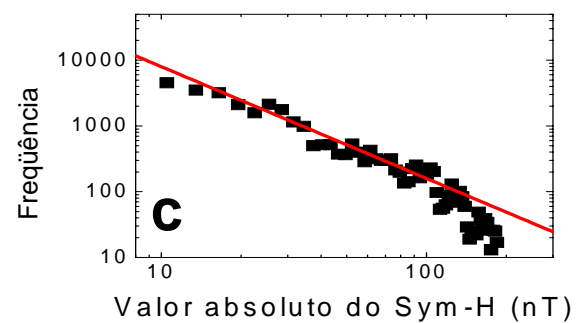

Figura 2 - Distribuição dos valores do índice Sym-H para o mês de outubro de 2000. Apresenta um regime de lei de potências com inclinação negativa.

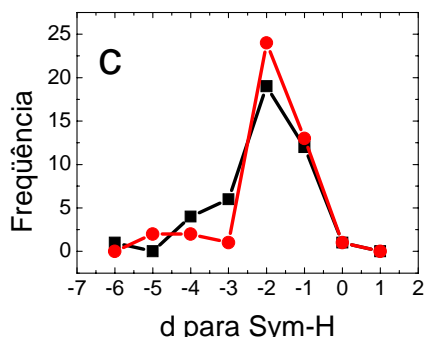

Figura 3 - Distribuições de inclinações, d, para Sym-H. Estão representados os dados para períodos imediatamente antes das tormentas (círculos vermelhos) e para períodos três dias antes das tormentas (quadrados pretos). Este convênio será valido em todas as figuras com duas cores.

A nossa análise foi feita sobre períodos de três dias enquanto que as dependências na Figura 2 foram obtidas com os dados correspondentes a um mês. Este foi o motivo para uma redução extra no número de tormentas efetivamente estudadas. Uma vez obtida a distribuição e calculada a inclinação (isto é, o expoente $d$ ) e o seu erro, foi estabelecido um limiar para o erro. Quando o erro foi maior que esse limiar a tormenta correspondente foi eliminada da estatística. O limiar foi de $30 \%$. Com isto, o número de tormentas efetivamente usadas foi 43.

A Figura 3 mostra a distribuição dos valores de $d$ para o Sym-H. Não existem diferenças estatísticas notáveis entre as distribuições para períodos próximos às tormentas e para períodos afastados destas (como comprovado por testes realizados). Que não existam diferenças entre os dois tipos de períodos podia ter sido esperado de resultados prévios [Wanliss, 2005; Papa et al., 2006] sobre o caráter fractal dessas distribuições: elas são, em princípio, indistinguíveis.

A dependência temporal de $d$ para Sym-H é mostrada na Figura 4a. Enquanto não há uma correspondência um a um entre as inclinações correspondentes a uma única tormenta, pode se notar que existe uma tendência de longo prazo (decrescendo com o tempo). Isto pode ser parcialmente provado qualitativamente observando a Figura $4 b$, onde se nota uma concentração preferencial de pontos ao redor da reta $y=x$.
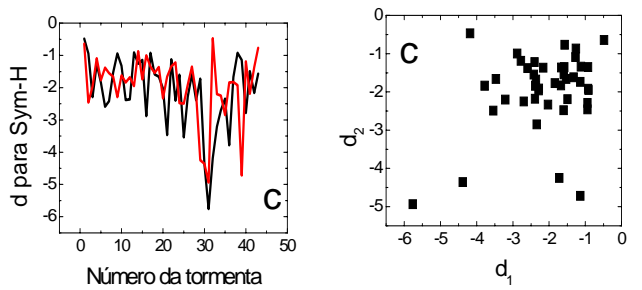

Figura 4 - a) Dependência temporal de d (Sym-H). b) Correlação das inclinações imediatamente antes das tormentas $\left(d_{2}\right)$ e três dias antes destas $\left(d_{1}\right)$.

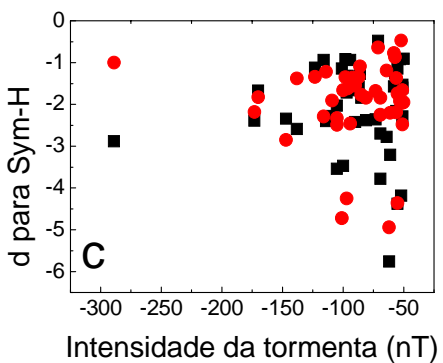

Figura 5 - Dependências das inclinações nas distribuições do índice Sym-H em função da intensidade de tormenta correspondente. Notar que parece existir uma região proibida na parte inferior esquerda do gráfico.

A análise apresentada até agora não envolveu a possibilidade de previsão de tormentas e sim apenas a demonstração de que existem mudanças efetivas de um tipo de período para o outro. Passamos agora ao estudo das possibilidades de previsão. Na Figura 5 é mostrada a dependência da inclinação, nos dois tipos de períodos, na intensidade da tormenta correspondente. Apesar de que temos uma estatística pequena, observamos, uma região proibida nesse gráfico. Essa região permite fazer afirmações do tipo seguinte: podemos dizer que se o expoente da lei de potências for menor que -3.5 então a 
tormenta seguinte não será mais intensa do que -125 nT (para qualquer um dos períodos).

\section{Medições do Observatório de Vassouras}

As medições com as quais trabalhamos neste item foram obtidas no Observatório Magnético de Vassouras. Estas medições são feitas a cada minuto e a incerteza do magnetômetro utilizado é de $1 \mathrm{nT}$. Os resultados que apresentamos correspondem também ao período de janeiro de 1998 até dezembro de 2002.

Uma vez determinado a partir do índice Dst o conjunto de períodos de interesse (como mostrado numa seção anterior), nós aplicamos uma metodologia recentemente introduzida na literatura [Papa et al., 2006] nos dois tipos de janelas temporais mencionadas anteriormente, isto é, os três dias imediatamente anteriores ao começo da tormenta e os três dias imediatamente anteriores a estes. Em poucas palavras, o método consiste em aplicar uma transformada de Fourier aos dados selecionados, filtrando estes com um filtro de Butterwoth de segunda ordem, retornando posteriormente a transformada ao domínio do tempo (transformada inversa) e, finalmente, calcular a diferença $\Delta \mathrm{H}$ entre os sinais original e filtrado (ver o trabalho por Papa et al. [2006] para uma descrição mais detalhada). A distribuição de $\Delta \mathrm{H}$ é o objetivo de estudo.

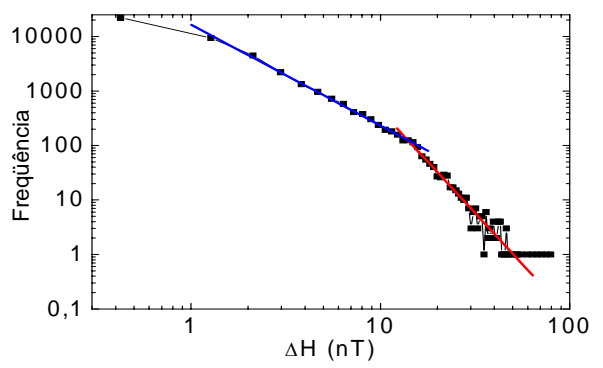

Figura 6 - Distribuição dos valores de $\Delta H$ para o mês de outubro de 2000.

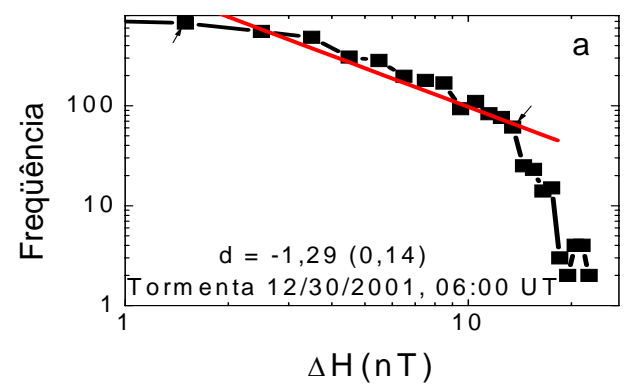

Figura 7 - Distribuição dos valores de $\Delta H$ para os três dias imediatamente anteriores à tormenta de 30/12/2001.

Quando esta metodologia é aplicada a períodos de tempo relativamente longos (um mês, por exemplo) são obtidas distribuições para $\Delta \mathrm{H}$ do tipo mostrado na Figura 6. Elas apresentam um duplo regime de leis de potências como descrito pela Equação (1) com um cotovelo entre os dois regimes sempre em valores entre 10 e $20 \mathrm{nT}$. Aplicar a metodologia a períodos de três dias, aqui também, é uma estratégia perigosa pois ela diminui dramaticamente (por um fator 10) o número de dados (piorando, conseqüentemente, a estatística). Um resultado típico para um período de três dias é mostrado na Figura 7. Agora, o segundo regime de lei de potências praticamente desapareceu. Nossa análise foi realizada sobre o primeiro regime ainda presente. Como conseqüência da pior estatística estabelecemos aqui também um limiar para o erro admissível nas inclinações. Inclinações que apresentaram erro relativo maior que $20 \%$ foram eliminadas da nossa estatística.

Antes de apresentar os resultados desta parte do trabalho gostaríamos de fazer alguns comentários sobre a análise e preparação de dados. Devido ao comportamento altamente irregular do índice Dst não foi possível estabelecer um procedimento computacional automático (livre da intervenção do homem) para selecionar os períodos a ser estudados. Neste sentido os resultados aqui apresentados são um produto "feito a mão". Eles foram analisados caso a caso individualmente. Uma situação similar foi enfrentada quando analisamos as inclinações das distribuições de $\Delta \mathrm{H}$. Isto pode ser um fator que dificulte a extensão deste tipo de estudo a períodos mais longos e possíveis estudos com propósitos de validação dos resultados aqui apresentados.

\section{Análise e Discussão}

É bem conhecido que as distribuições das medições do campo magnético na superfície da Terra [Papa et al., 2006] assim como as de alguns índices magnéticos [Wanliss, 2005] seguem leis de potência ou, em outras palavras, são compatíveis com algum sistema fractal. $\mathrm{Na}$ Figura 8a apresentamos a dependência temporal de $d$ nas distribuições para $\Delta \mathrm{H}$ no período 1998-2002.

Como pode ser visto na Figura $8 a$, enquanto não existe uma correlação um a um entre as inclinações para os períodos próximos às tormentas e aqueles longe das tormentas, parece existir (como para o índice Sym-H) uma tendência de longo prazo (aumentando com o tempo) nos dois tipos de inclinação. Estes resultados podem ser qualitativa e parcialmente comprovados na Figura $8 b$. onde se nota, novamente, uma concentração ao redor da reta $y=x$ (independentemente da escala utilizada).

A Figura 9 mostra as distribuições de inclinação para ambos tipos de períodos de três dias durante os anos de 1998 até 2002, para $\Delta \mathrm{H}$. Ela mostra uma tendência para valores maiores para os períodos mais próximos das tormentas. Essa tendência pode ser evidência de componentes não auto-similares (isto é, não fractais) nos registros magnéticos, o que significa que a previsão efetiva em médio prazo pode ser uma tarefa não tão dificultosa como imaginado até agora.

Finalmente apresentamos na Figura 10 a dependência das inclinações na intensidade da tormenta correspondente. Uma região proibida bem marcada está presente. Regiões proibidas são úteis para o propósito de previsão já que, mesmo se elas não conseguem dizer o que vai a acontecer, elas conseguem nos informar sobre o que não vai a acontecer ou, ainda, a probabilidade de que um certo evento aconteça. 

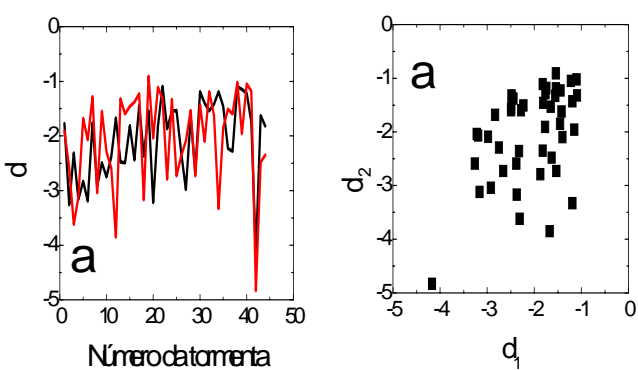

Figura 8 - a) Dependência temporal de $d(\Delta H)$. b) Correlações entre as inclinações imediatamente antes das tormentas $\left(d_{2}\right)$ e três dias antes destas $\left(d_{1}\right)$, para $\Delta H$.

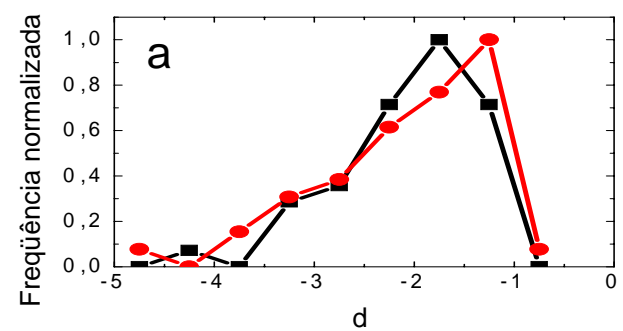

Figura 9 - Distribuições das inclinações no período 19982002 para $\Delta H$.

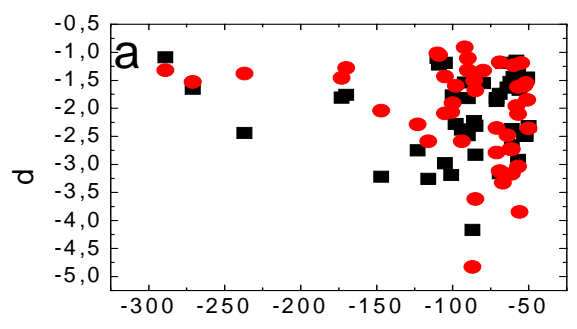

Intensidade da tormenta ( $\mathrm{nT}$ )

Figura 10 - Dependências das inclinações na distribuição de $\Delta H$ em função da intensidade de tormenta correspondente. Notar que parece existir uma região proibida.

Pode se deduzir da Figura 10 que se a inclinação para um período próximo de alguma tormenta tiver um valor menor que $-2,5$, então a próxima tormenta jamais terá intensidade maior que $-150 \mathrm{nT}$. Da mesma figura que, se a inclinação de um período próximo estiver entre $-1,75$ e $-1,25$, então existe uma probabilidade de ao redor de $30 \%$ para que a próxima tormenta seja mais intensa que -150 nT. O valor de $30 \%$ foi obtido notando que existem cinco pontos (círculos) no retângulo formado pelos valores $d=-1,75$ e $-1,25$ e intensidade da tormenta $=-$ $150 \mathrm{nT}$ e $-325 \mathrm{nT}$, enquanto existem dez pontos (círculos também) no retângulo formado pelos mesmos valores de $d$ e intensidade da tormenta $=-150 \mathrm{nT}$ e $-50 \mathrm{nT}$.

Deve ser notado também na Figura 10 que, dado que não existem distribuições com valores maiores que $-0,9$ para a inclinação, os períodos próximos das tormentas definem uma região proibida mais restritiva (círculos) que aquela definida pelos períodos mais afastados das tormentas (quadrados). Isto é intuitivamente claro se acreditamos, como parece ser o caso, que existe efetivamente alguma mudança nas distribuições de inclinação de um tipo de período em relação ao outro: quanto mais próximo o período em estudo estiver da tormenta, mais detalhada e precisa será a informação que ele pode oferecer sobre a própria tormenta.

\section{Conclusões}

Foi demonstrada, através do estudo de algumas propriedades fractais do índice Sym-H (para aplicações globais) e de medições geomagnéticas diretas (com vista à sua aplicação local), a possibilidade de previsão probabilística da intensidade do campo magnético nos períodos de maior perturbação. Como mostrado em trabalhos anteriores, esta ocorrência está correlacionada às maiores variações na declinação e na inclinação magnéticas. Estes fatos abrem a possibilidade de utilização destes dados nas atividades de prospecção e perfuração a ser realizadas. Espera-se que, devido à proximidade, os dados do Observatório Magnético de Vassouras possam vir a ser especialmente úteis nas atividades desse tipo a ser desenvolvidas nos estados de $B A, E S, M G, R J$ e SP. Regiões mais para o norte poderão se beneficiar de estudos similares realizados a partir de medições obtidas no Observatório de Tatuoca (PA).

\section{Referências}

Gleisner, H.; Rasmussen O.; Watermann, J., 2005, Large-magnitude geomagnetic disturbances in the North Sea region: Statistics, causes, and forecasting, Adv. Space Res., doi:10.1016/j.asr.2005.04.082, e suas referências.

Halberg, F.; Cornélissen, G.; Otsuka, K.; Katinas, G.; Schwartzkopff O., 2001, Essays on Chronomics Spawned by Transdisciplinary Chronobiology. Witness in Time: Earl Elmer Bakken, Neuroendocrinology Letters 22, 359-384.

Jansen, F.; Munakata, K.; Duldig, M. L.; Hippler, R., 2001, Muon Detectors - The Real-Time, Ground Based Forecast of Geomagnetic Storms in Europe, Proceedings of the Space Weather Workshop: Looking Towards a European Space Weather Programme, 17-19 December 2001, ESTEC, Noordwijk, The Netherlands.

Maltsev, Y. P., 2003, The Points of Controversy in Magnetic Storm Study (Review), Physics of Auroral Phenomena, Proc. XXVI Annual Seminar, Apatity, 33-40, Kola Science Center, Russian Academy of Sciences.

Papa, A. R. R.; Barreto, L. M.; Seixas, N. A. B., 2006, Statistical Study of Magnetic Disturbances at the Earth's Surface, Journal of Atmospheric and Solar Terrestrial Physics 68, 930-936.

Wanliss, J., 2005. Fractal properties of SYM-H during quiet and active times. Journal of Geophysical Research 110, A03202.

Yermolaev Y. I.; Yermolaev, M. Y., 2006, Geomagnetic Storm Dependence on the Solar Flare Class, arXiv:physics/0601197 v1, e referências. 